\title{
Non-Linear Non-Destructive Evaluation of Concrete
}

\author{
A.A. Shah ${ }^{1}$ and Y. Ribakov ${ }^{2, *}$ \\ ${ }^{I}$ Department of Mechanical and Environmental Informatics, Tokyo Institute of Technology, Japan; ${ }^{2}$ Department of Civil \\ Engineering, Ariel University Center of Samaria, Ariel, Israel
}

\begin{abstract}
This paper explains non-linear non-destructive evaluation of concrete aimed at examining the occurrence of non-linearity under non-linear wave propagation through the concrete. Standard cubic concrete sections in damaged and undamaged conditions were investigated. The specimen was first damaged under compression testing and then subjected to non-linear ultrasonic evaluation in two directions i.e., perpendicular and parallel to the direction of compression loading. For observing non-linearity the ultrasonic pulse was transmitted at high power level through $100 \mathrm{kHz}$ broadband frequency transducer. The obtained test results for the concrete cube both in damaged and undamaged conditions were plotted and compared. The ratios of harmonic generations in decibels were calculated and plotted as normalized frequency spectrums. It was shown that the wave propagation in damaged specimen in a direction perpendicular to compression loading is more sensitive to cracking in concrete than in a direction parallel to compression loading.
\end{abstract}

Keywords: Non-linear ultrasonic; damaged concrete; broadband; time domain; fast fourier transform; frequency domain.

\section{INTRODUCTION}

Stress wave propagation methods have been often used in non-destructive testing (NDT) of concrete. Features concerning strength, porosity and damage of concrete can be adequately revealed by analyzing ultrasonic signals $[1,2]$. Due to the highly inhomogeneous nature of the material though, consisting of cement, sand, fine and coarse aggregates, water and air bubbles, results are usually valid for qualitative only conclusions, without direct correlation with structural integrity parameters. While, concrete structures are continually subject to effects that degrade their structural integrity. For example, damage from micro-cracking develops at length scales that are smaller than the size of the majority of the aggregate [3]. Such cracks start to appear in concrete in its very initial stages of hardening even when it is yet to be subjected to any load application.

Micro-cracking usually occurs at the interface of bond between the coarse aggregate and cement mortar. Under applied load, new micro-cracks form and the existing microcracks grow and propagate into larger cracks until failure is occurred [4]. The detection of such cracks or defects appears at early stages are therefore mandatory in saving the life of a concrete structure. Therefore, the possibility of performing defect detection at a micro level using a linear ultrasonic wave method has been examined in recent years. However, the use of traditional linear nondestructive testing methods in damage evaluation at early stages is not so effective [5]. Methods like ultrasonic pulse velocity and resonant frequency give little indication of the damage present in a con

*Address correspondence to this author at the Department of Civil Engineering, Ariel University Center of Samaria, Ariel, Israel; Tel: +972-546 431 385: Fax: +972 390663 51: E-mail: ribakov@ariel.ac.il crete structure until this damage progresses to the point of causing a major problem. Nonlinear ultrasound, on the other hand, has been shown to be extremely sensitive to early damage evaluation in concrete. This sensitivity extends from very low damage levels to very high damage levels corresponding to strain localization and fracture [6].

Furthermore, application to a solid material was considered to be difficult because a nonlinear ultrasonic wave is not easily generated in a solid material. However, a nondestructive inspection method using a nonlinear ultrasonic wave has begun to attract attention due to the recent advancements of a high power ultrasonic wave drive device.

When an ultrasonic wave is injected into a material, the ultrasonic waveform passing through the material corresponds to the stress-strain relationship. If an ultrasonic wave having small amplitude, like the stress-strain relationship is linear, the received ultrasonic waveform does not change compared with the incident ultrasonic waveform. However, if an ultrasonic wave having large amplitude, which arrives at the plastic modification domain of a material, is injected into the material, the waveform of the incident ultrasonic wave is considered to be distorted. As a result, the waveform of the penetrated ultrasonic wave is different from that of the injected ultrasonic wave. Therefore, in relation to the incident ultrasonic wave, the penetrated ultrasonic wave has harmonic frequency components [7].

In the present research the suitability of the non-linear ultrasonic method in defect detection has been shown. Ultrasonic wave is transmitted into the concrete section (damaged or undamaged) at power levels producing larger amplitude in order to examine the occurrence of non-linearity or harmonic amplitudes by measuring difference between the injected and penetrated waveforms, if any. 


\section{RESEARCH OBJECTIVES}

The primary objective of this research was to track harmonic amplitudes of the transmitted signal during testing the concrete cube both in damaged and undamaged conditions. For this purpose, results from an experimental study of through transmission non-linear ultrasonic measurements on cubic concrete specimen is described revealing interesting features concerning the influence of the wave propagation direction and the specimen's physical condition (damaged or undamaged) on detecting harmonic components of the propagating wave.

\section{EXPERIMENTAL PROGRAM}

The ultrasonic tests were performed on three cube concrete specimens (A1, A2, and A3) of $150 \times 150 \times 150 \mathrm{~mm}$ in size in both damaged and un-damaged conditions. The concrete mixture proportions are listed in Table 1. Type I Portland cement with $3.16 \mathrm{gm} / \mathrm{cm}^{3}$ density and locally available sand of $2.62 \mathrm{gm} / \mathrm{cm}^{3}$ density with absorption capacity of $1.50 \%$ were used in the concrete mix. Crushed stones of 2.62 $\mathrm{gm} / \mathrm{cm}^{3}$ density having absorption capacity of $0.65 \%$ were used as coarse aggregate and the maximum aggregate size was $12 \mathrm{~mm}$. All the specimens were cast with water to cement ratio (w/c) of $40 \%$. In addition, a high range water reducing admixture, air entraining agent and rod vibrator were used to improve workability and consolidation of concrete. Cubes were cast vertically on a level surface. Specimens were prepared in steel moulds with maximum vibration time of $15 \mathrm{~s}$. After casting all specimens were kept for moistcuring. The cubes were demoulded at $24 \mathrm{~h}$ age. After removal of the moulds they were stored in a standard moist room at a temperature of $20 \pm 3^{\circ} \mathrm{C}$ until the moment of ultrasonic test.

One of the above specimens (A2) was first damaged under compression before applying the non-linear ultrasonic measurement procedure. The axial compressive load to the specimen was applied using universal testing machine (UTM, closed loop servo-hydraulic testing machine) with a capacity of 200 tons using a displacement control method. The compression test was performed using a displacement control method of $0.003 \mathrm{~mm} / \mathrm{s}$ velocity. The rate was applied continuously and without shock. Load application was measured continuously using load cell until the ultimate level of the specimen is reached.

Table 1. Mix Design Proportions

\begin{tabular}{|c|c|}
\hline Item & Quantity $\left(\mathbf{k g} / \mathbf{m}^{\mathbf{3}}\right)$ \\
\hline \hline Cement & 50 \\
\hline Sand & 89 \\
\hline Aggregate & 102 \\
\hline Water & 20 \\
\hline Water Reducing Admixture & 0.144 \\
\hline Air Entraining Agent & 0.24 \\
\hline
\end{tabular}

The specimen's compressive strength measured at the end of the test was $50 \mathrm{MPa}$. After ultimately failed in com- pression, the specimen was unloaded and subjected to ultrasonic evaluation using non-linear ultrasonic technique (see Fig. 1). The remaining two specimens (A1, \& A3) were evaluated ultrasonically in the undamaged conditions. Due to similarities in behavior of the undamaged specimens A1 and $\mathrm{A} 3$, test results of only A1 are presented in this research.

A non-linear ultrasonic test setup was used that created an ultrasonic signal of $100 \mathrm{kHz}$ frequency varied over a voltage range between 100 and $400 \mathrm{~V}$. The center frequency of the receiving transducer was kept as $300 \mathrm{kHz}$. The test setup consists of a broadband square pulser, a receiver, two transducers: one of $100 \mathrm{kHz}$ broadband for signal transmission while the other of $500 \mathrm{kHz}$ broadband for signal receiving, and an oscilloscope (see Fig. 1). In testing the concrete cube both in damaged and undamaged conditions, as mentioned, tracking harmonic amplitudes of the transmitted signal was the primary goal.

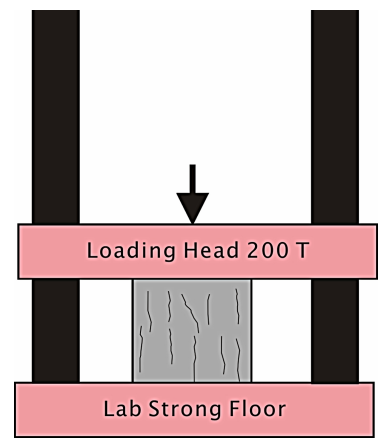

(a)

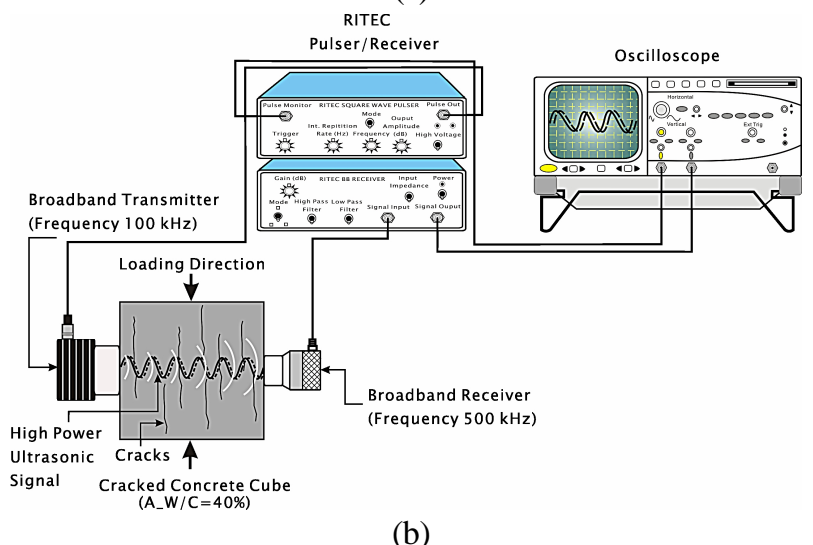

(b)

Fig. (1). Testing plan (a) compression testing and (b) non-linear ultrasonic testing.

Firstly the specimens in the undamaged conditions were ultrasonically examined and the obtained waveforms were then plotted in time and frequency domains. Furthermore, the normalized frequency plots were prepared from which the ratios of harmonic generations were measured and expressed in decibels. These results were then compared with those obtained from the test of the same but damaged specimen. As it was mentioned above, the damages to such specimen were induced under compression ultimate loading.

It is noted here, that the damaged concrete specimen was ultrasonically evaluated chiefly in a direction perpendicular to the compressive loading (see Fig. 1). Later for encountering any cracks by the transmitted waves the same specimen was also evaluated ultrasonically parallel to the loading di- 
rection. Like the undamaged specimens the data obtained was plotted both in time and frequency domains. The harmonic amplitudes were measured and the change in fundamental amplitude was calculated.

\section{RESULTS AND DISCUSSION}

Fig. (2) shows the time and frequency domain curves for the concrete cube (A1) in undamaged state. These waveforms, as stated earlier, were obtained using a $100 \mathrm{kHz}$ broadband transducer as transmitter and a $500 \mathrm{kHz}$ one as receiver. The concrete cube was ultrasonically evaluated in two directions. The wave propagation in second direction was conducted by rotating the concrete cube at 90 degrees to the first one. The signals of $100 \mathrm{kHz}$ frequency were transmitted into the cube at the voltage level of $100 \mathrm{~V}$.

In the frequency spectrum the occurrence of second harmonic components, when the fundamental frequency peak was passed, is clearly visible. The wave propagation thus detected the presence of micro-crack or defect, possibly appeared due to curing or shrinkage in the interfacial transition zone, even when there was no load application to the specimen. However, the amplitude levels in each plot obtained during ultrasonic testing of undamaged specimen in both directions are closely comparable, although the second direction fundamental amplitude peak is slightly lower than the first one. This can be because the wave may have encountered slightly large percentage of voids while propagating in that particular direction and hence led to a slight decrease in the amplitude level.

The waveforms in Fig. (2b) were normalized at peak amplitude and a normalized frequency plot was prepared calculating the amplitude values in decibels as:

$$
\text { Amplitude }[\mathrm{dB}]=-20 \log \left(\frac{A_{1}}{A_{0}}\right)
$$

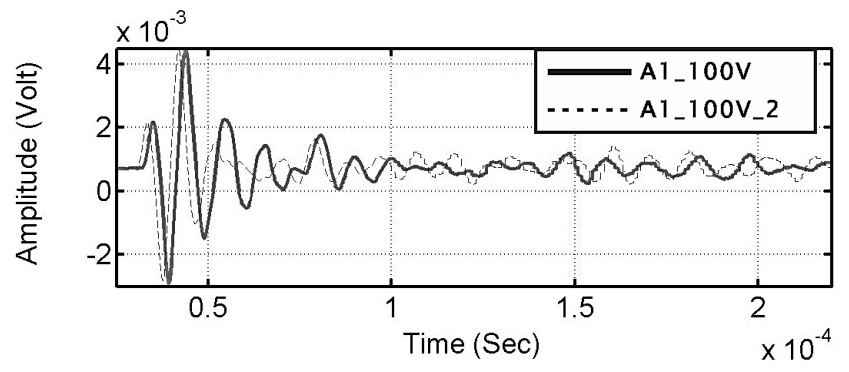

(a)

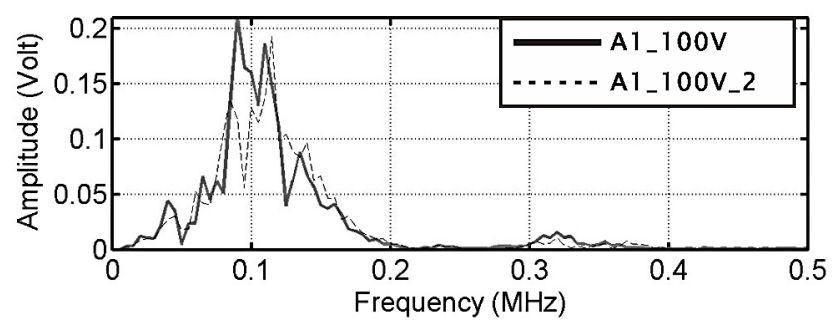

(b)

Fig. (2). Waveforms for undamaged concrete cube at $100 \mathrm{~V}$ (a) time domain, and (b) frequency domain.
Parameter $A_{0}$ is the reference amplitude and $A_{1}$ is the amplitude, which is compared with the reference amplitude.

Fig. (3) shows the amplitude vs. frequency graph. The amplitude values are calculated and expressed in decibels (Eq. 1). These values appeared to be almost similar in both directions, as in Fig. (2) as well. Contrary to the first peak appeared at $100 \mathrm{kHz}$ frequency the second harmonic peak that appeared at $300 \mathrm{kHz}$ is reduced by about $24 \mathrm{~dB}$.

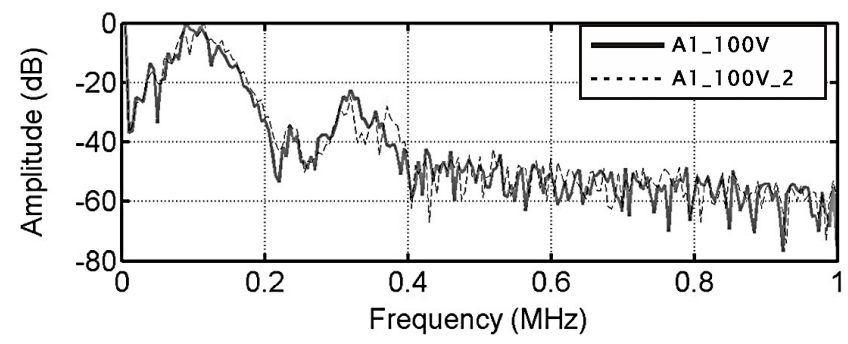

Fig. (3). Normalized frequency plot for undamaged concrete cube at $100 \mathrm{~V}$.

Fig. (4) shows the plots for the same specimen (A1) evaluated by generating the ultrasonic pulse at $400 \mathrm{~V}$. Increasing the voltage level yields an increase in amplitudes. The frequency domain graph appeared into the fundamental frequency peak and the second or higher harmonic peak, showing the tracking of harmonic components by the nonlinear wave propagation through the concrete.

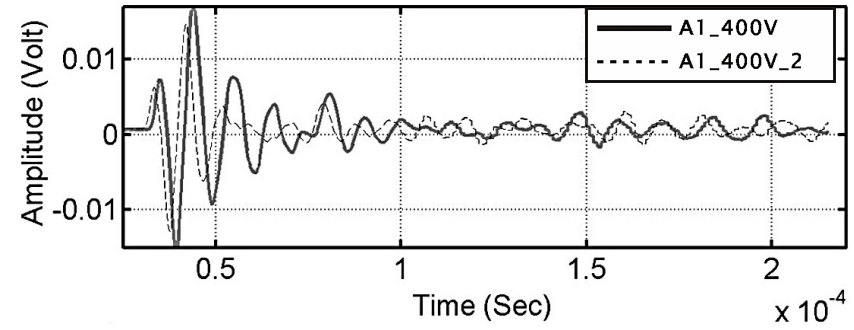

(a)

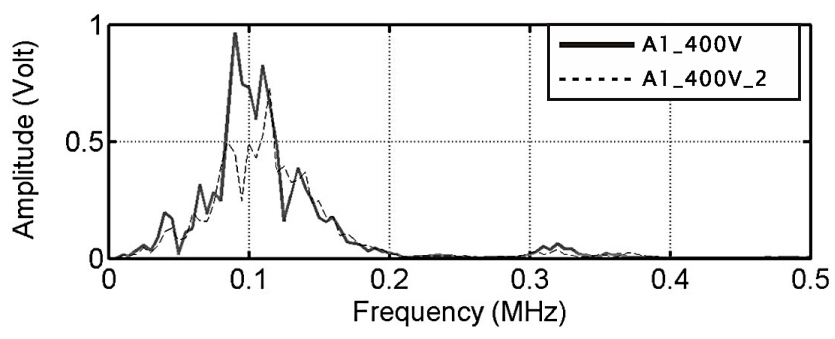

(b)

Fig. (4). Waveforms for undamaged concrete cube at $400 \mathrm{~V}$ (a) time domain, and (b) frequency domain.

Once again the frequency domain waveforms were used in comparing the amplitudes with the reference amplitude of the peak level and calculate the values in decibels (Eq. 1). The calculated values are plotted against the frequency values, as shown in Fig. (5). Though somewhat higher values than those in Fig. (3), which are obviously due to transmitting the pulse at higher voltage, almost the same trend is visible from this plot. The difference between the two frequency peaks appeared as $24 \mathrm{~dB}$. 


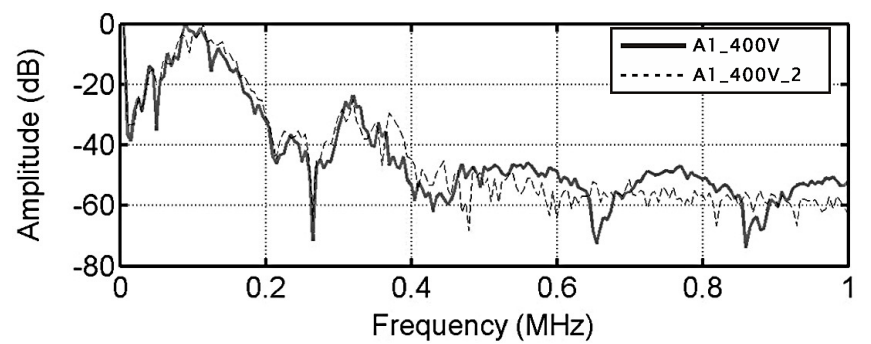

Fig. (5). Normalized frequency plot for undamaged concrete cube at $400 \mathrm{~V}$.

For the specimen in damaged conditions (A2) the plots are given in Fig. (6). The specimen was ultrasonically evaluated both in directions parallel and perpendicular to compression loading. The pulse generation was made at $100 \mathrm{~V}$, while the transmitted signal to drive the transmitter had a driving frequency of $100 \mathrm{kHz}$.

Upon looking into the waveforms, the amplitude level in direction parallel to compression loading appeared to be higher than that of perpendicular one. This is because most cracks appear on concrete face parallel to the load application under compression. Wave propagation through such direction, therefore, may miss encountering cracks. As a result the amplitude level was higher in this case, which decreases as the ultrasonic wave passes through a crack. On the other hand in case of perpendicular ultrasonic evaluation, the transmitting signals have many chances of passing through the cracks, which leads to higher harmonic generations and reduction in the amplitude levels.

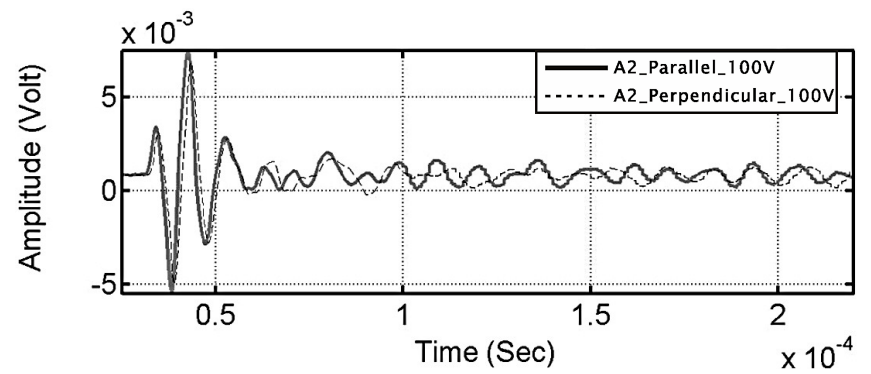

(a)

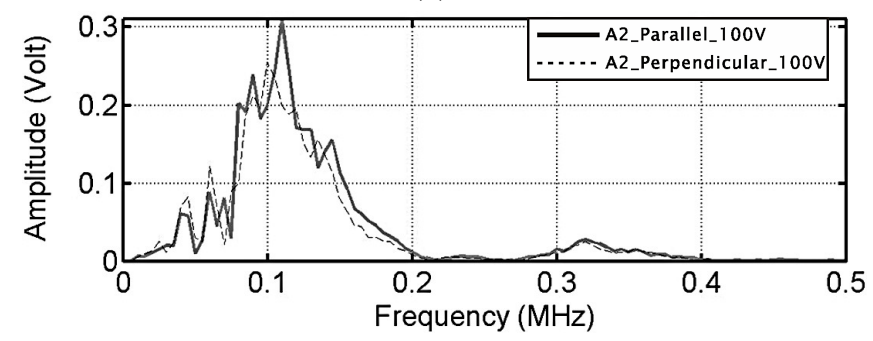

(b)

Fig. (6). Waveforms for damaged concrete cube at $100 \mathrm{~V}$ (a) time domain, and (b) frequency domain.

Fig. (7) illustrates the amplitude values in decibels for the same specimen. The values in both parallel and perpendicular directions to the compression loading although are similar. Slightly higher peak of the second harmonic is obtained in Fig. (6b) than that in Fig. (2b). This resulted into the difference between the harmonic frequency peaks at 100 and $300 \mathrm{kHz}$ as $20 \mathrm{~dB}$. Comparison of this value with that ob- tained in the undamaged case (Fig. 2) show the occurrence of higher harmonic wave components during wave propagation through a damaged concrete section over an undamaged concrete section. This small change in the amplitude values of damaged and undamaged specimens can also be considered as a sign of the presence of non-linearity in a cracked specimen.

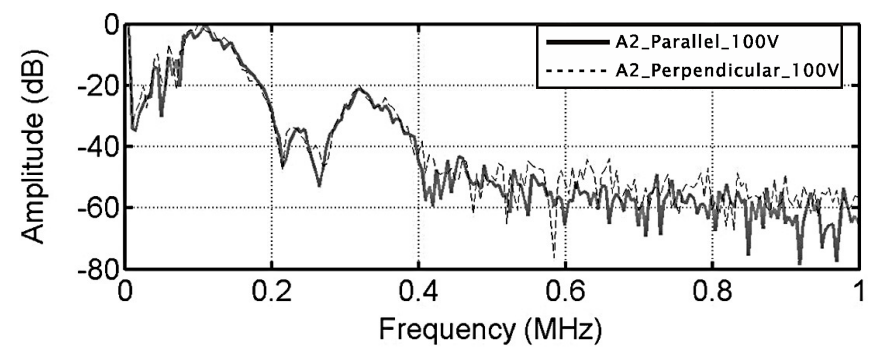

Fig. (7). Normalized frequency plot for damaged concrete cube at $100 \mathrm{~V}$.

Similar to Figs. (6) and (7), Figs. (8) and (9) show the time vs. amplitude and frequency vs. amplitude graphs for the same specimen evaluated with signal transmission at 400 V. Like in Fig. (6), in Fig. (8) the amplitude values in a direction parallel to loading are mostly greater than those obtained with wave propagation perpendicular to loading. The reason, as stated earlier, is the presence of fewer numbers of cracks in wave propagation direction parallel to compression loading. Wave propagation in perpendicular direction to compression loading, however, has enough cracks to encounter in a cracked section and therefore showed increased sensitivity to damage over the case where wave propagation direction was parallel to compression load application.

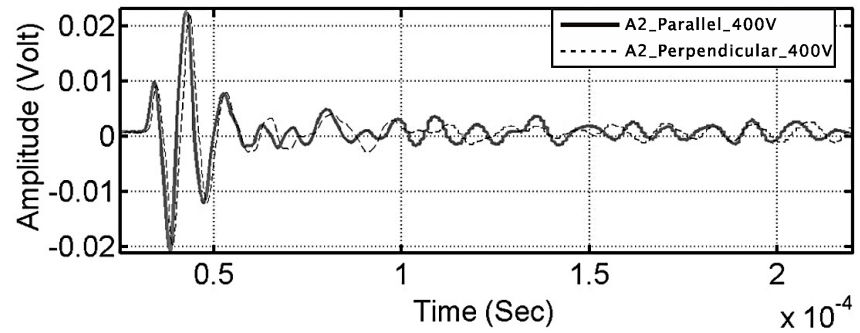

(a)

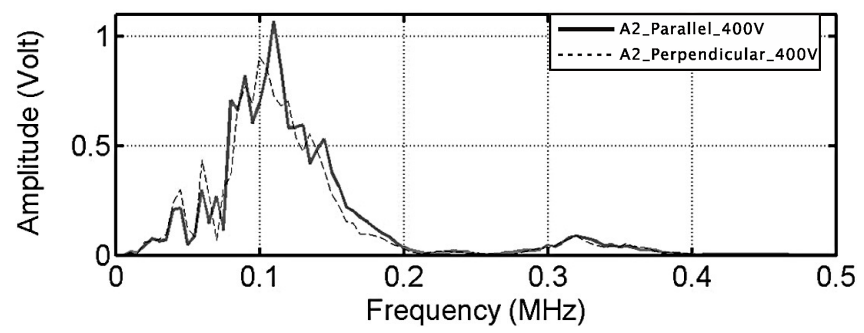

(b)

Fig. (8). Waveforms for damaged concrete cube at $400 \mathrm{~V}$ (a) time domain, and (b) frequency domain.

A normalized frequency plot showing the change in amplitude values in decibels is given in Fig. (9). Again the change in the two amplitude peaks is counted as $20 \mathrm{~dB}$, which is $4 \mathrm{~dB}$ less than what is obtained in testing the undamaged specimen (see Fig. 3 or Fig. 5). Hence the signs of occurrence of higher harmonics, compared to the results of undamaged specimen, are also evident from this plot. 


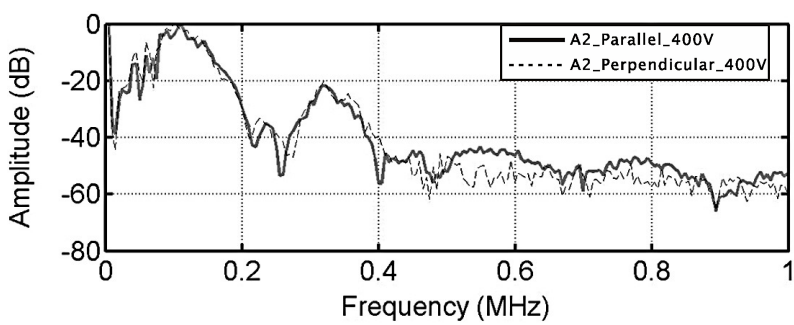

Fig. (9). Normalized frequency plot for damaged concrete cube at $400 \mathrm{~V}$.

In view of the above details, one can see very small change in the amplitude values of damaged and undamaged cases. This is due to the fact that low water cement ratio concrete has fewer defects in the form of voids and porosity as well as a stronger interfacial transition zone than higher water cement ratio concrete has. Increase in such defects may lead to premature micro-crack formation and increased attenuation, which was therefore not the case in ultrasonic testing of $40 \% \mathrm{w} / \mathrm{c}$ concrete and can be expected in testing higher w/c $(50 \%$ or $60 \%)$ concretes. Thus, the change in amplitude for w/c of $40 \%$ was not so high.

\section{CONCLUSIONS}

The present research was aimed at evaluating the cube concrete specimens in damaged and undamaged conditions under non-linear ultrasonic testing. The damages were induced into the concrete specimen by testing it under compression loading up to its ultimate capacity. In damaged or undamaged case the test specimen was ultrasonically evaluated in two directions. The undamaged specimen tested in two directions yielded almost similar results. In case of the damaged specimen the wave propagation direction was designated as perpendicular and parallel to compression loading. It was found from the analysis of the test results that the wave propagation in a direction perpendicular to compression loading is more sensitive to cracking in concrete than in a direction parallel to compression loading. The change in frequency peaks of the damaged specimen was observed smaller $(20 \mathrm{~dB})$ than that of the undamaged specimen $(24$ $\mathrm{dB}$ ), which means the occurrence of higher harmonics in damaged specimen over undamaged specimen. This also shows the presence of non-linearity that occurred when the wave propagated through the cracked concrete section.

The method presented in this paper has been found effective for detecting micro-cracks. It has the advantage that unlike linear ultrasonic measurement it generates higher harmonics while propagating through a fatigued material and therefore it can be recommended as a useful tool in rehabilitation of concrete structures or structural health monitoring.

\section{ACKNOWLEDGEMENT}

The research grant for conducting this research was provided by Japan Society for the Promotion of Science (JSPS), which is thankfully acknowledged.

\section{REFERENCES}

[1] V. M. Malhotra and N. J. Carino, Eds., CRC handbook on nondestructive testing of concrete, Florida: CRC Press, 1991.

[2] T. Umoto. Ed., Non-destructive testing in civil engineering, Amsterdam: Elsevier, 2000.

[3] S. Popovics and J. Popovics, "Ultrasonic testing to determine water-cement ration for fresh concrete", American Standard for Testing and Materials, vol. 20, pp. 262-268, 1998.

[4] D. J. Stauffer, C. Woodward and K. R.White, "Non-linear ultrasonic testing with resonant and pulse velocity parameters for early damage in concrete", ACI Materials Journal, vol. 102(2), pp. 118121, 2005.

[5] K. Y. Jhang, "Applications of nonlinear ultrasonics to the NDE of materials degradation", IEEE Transactions on Ultrasonics, Ferroelectrics, and Frequency Control, vol. 47(3), pp. 540-548, 2000.

[6] C. Woodward, S. E. Roe and M. Cramer, "Ultrasonic attenuation changes with damage level in concrete", in Review of progress in quantitative non-destructive evaluation, D. O. Thompson and D. E. Chimenti, Eds., Springer, vol. 894, pp. 1340-1344, 2007.

[7] R. Murayama and K. Yamaguchi, "Non-linear ultrasonic wave detection by electromagnetic acoustic transducer [EMAT] for guided wave", Japanese Journal of Applied Physics, vol. 44(6B), pp. 4385-4388, 2005. 